\title{
CMEARTICLE
}

\section{Clinics in diagnostic imaging (174)}

Manickam Subramanian ${ }^{1}$, FRCR, Hong $\underline{C h o u}^{2}$, FRCR, Kabilan $\underline{\text { Chokkappan }}^{1}$, FRCR, Wilfred CG Peh${ }^{2}$, FRCP, FRCR
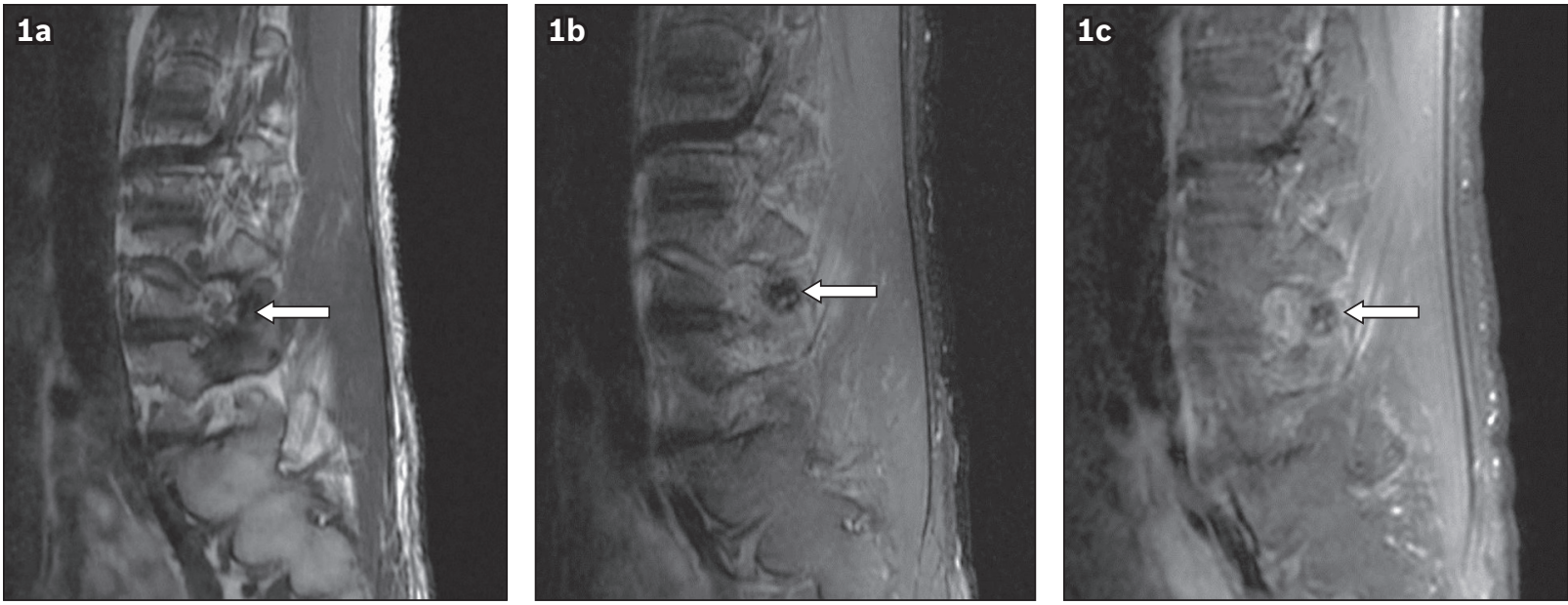

Fig. 1 Right parasagittal (a) T1-W turbo spin-echo (TSE), (b) T2-W TSE and (c) contrast-enhanced fat-suppressed T1-W TSE MR images of the lower lumbar spine.
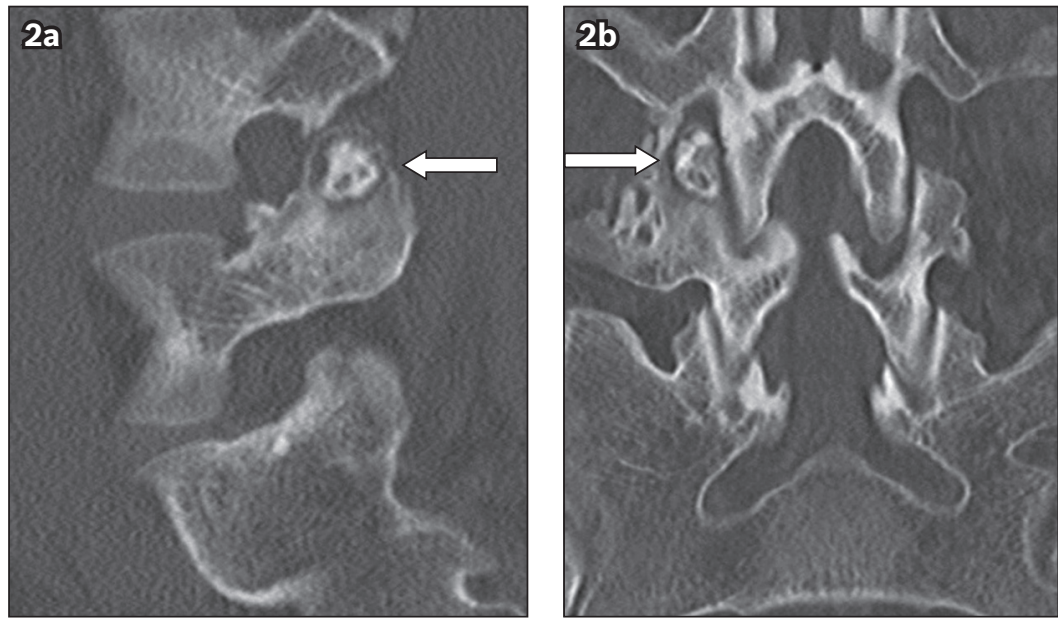

Fig. 2 Right (a) parasagittal and (b) coronal reformatted CT images of the lower lumbar spine.

\section{CASE PRESENTATION}

A 25-year-old man presented with chronic low back pain that occasionally radiated to the right lower limb. The low back pain was a constant, dull ache and had been present for one year. No motor weakness, sensory deficit, bowel or bladder disturbance was noted. There was no worsening of pain at night. On physical examination, focal tenderness was noted at the L5 vertebral level. The patient's physical examination was otherwise normal. Routine blood investigations did not reveal any abnormality. Magnetic resonance (MR) imaging (Fig. 1) followed by computed tomography (CT) of the lumbar spine (Fig. 2) was performed. What do these images show? What is the diagnosis?

'Department of Diagnostic Radiology, Tan Tock Seng Hospital, ${ }^{2}$ Department of Diagnostic Radiology, Khoo Teck Puat Hospital, Singapore

Correspondence: Dr Manickam Subramanian, Radiology Resident, Department of Diagnostic Radiology, Tan Tock Seng Hospital, 11 Jalan Tan Tock Seng, Singapore 308433. spmanickam@yahoo.com 

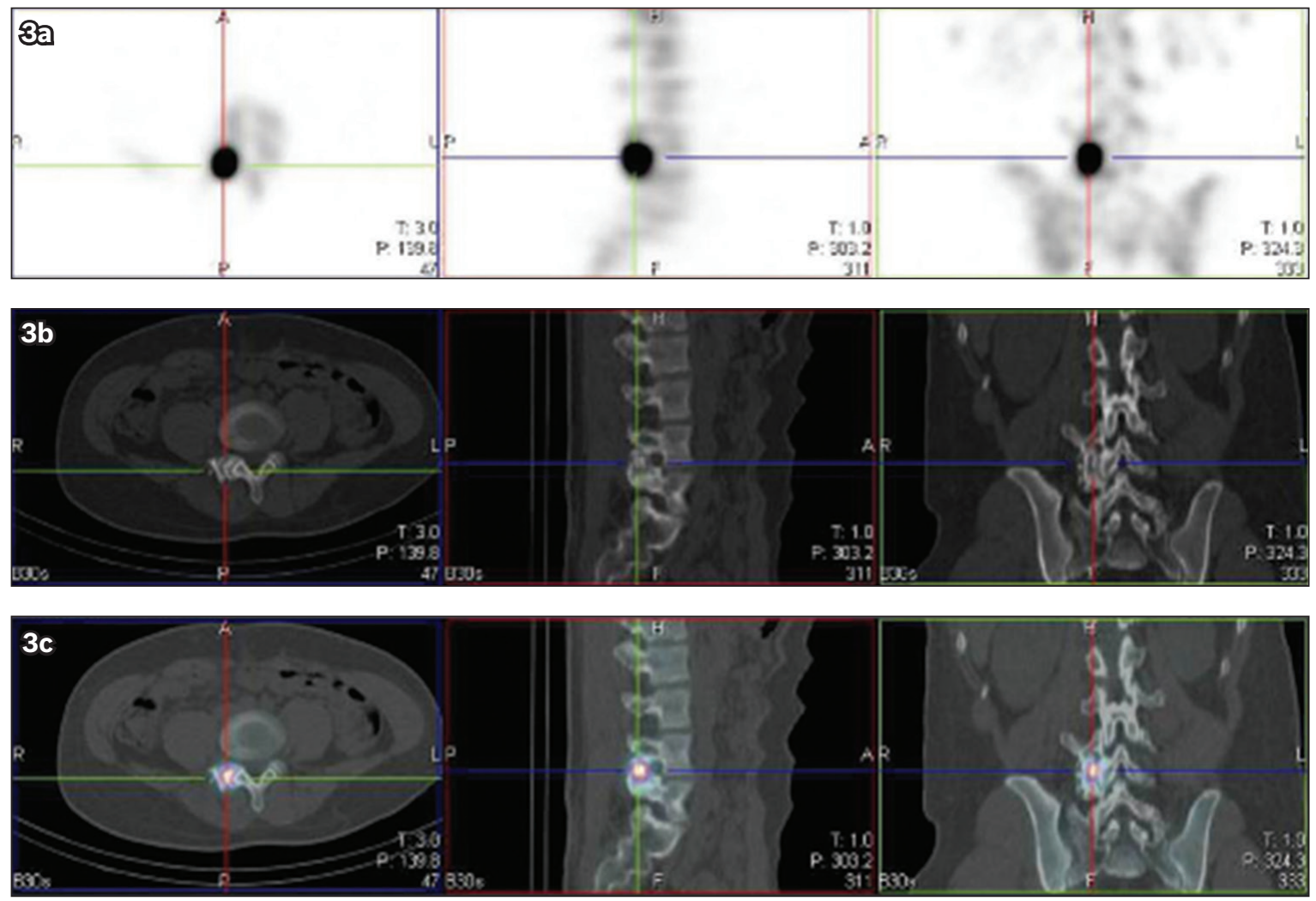

Fig. 3 (a) Single-photon emission CT (SPECT), (b) CT and (c) combined SPECT/CT images of the lower lumbar spine in three orthogonal planes show that increased tracer uptake corresponds to the osteolytic expansile lesion with sclerotic nidus in the right superior facet of the $L 5$ vertebra.

\section{IMAGE INTERPRETATION}

MR imaging of the lumbosacral spine showed a well-defined lesion, which was hypointense on T1- and T2-weighted turbo spin-echo imaging, in the right superior facet of the L5 vertebra (arrow) with surrounding bone marrow and soft tissue oedema (Figs. 1a \& b). There was mild rim enhancement of the lesion and surrounding soft tissues (Fig. 1c). These inflammatory soft tissue changes also extended toward the right neural foramen. CT of the lumbar spine (Figs. 2a \& b) showed a well-defined osteolytic expansile lesion with a sclerotic nidus (arrows) measuring $2.2 \mathrm{~cm}$ $($ craniocaudal $) \times 1.7 \mathrm{~cm}$ (anteroposterior) $\times 1.4 \mathrm{~cm}$ (transverse) in the right superior facet of the L 5 vertebra, with cortical irregularity and secondary bony expansion in the right pedicle and facet of the L5 vertebra.

\section{DIAGNOSIS}

L5 vertebral superior facet osteoblastoma $(\mathrm{OB})$.

\section{CLINICAL COURSE}

The patient underwent triple-phase technetium-99m methylene diphosphonate (Tc-99m MDP) bone imaging and single-photon emission CT/CT (SPECT/CT) of the lumbosacral spine. Triplephase Tc-99m MDP imaging showed increased blood-pool tracer accumulation in the lower right lumbar spine in the equilibrium phase. In delayed imaging, this focus of increased tracer uptake had an oval pattern and was localised to the right superior articular facet of the L5 vertebra on SPECT/CT (Fig. 3).

CT-guided biopsy and radiofrequency ablation (RFA) of the lesion was performed under general and local anaesthesia.
A 22-gauge spinal needle was placed into the right L4 nerve root sleeve via the parafacetal approach. Nerve root block was administered using a mix of $1 \mathrm{~mL}$ triamcinolone $40 \mathrm{mg}$ and $2 \mathrm{~mL}$ $0.5 \%$ bupivacaine. The needle was left in place and connected to chilled dextrose $5 \%$ infusion for neuroprotection. Biopsy was performed using a 14.5-gauge Ostycut needle (Fig. 4). A 1.5-cm core was obtained with a single pass. The RFA needle $(1 \mathrm{~cm}$, non-cooled active tip) was introduced along the same tract and two cycles of six-minute RFA ablation was performed with a target temperature of $90^{\circ} \mathrm{C}$ (Fig. 5 ). $2 \mathrm{~mL} 0.5 \%$ bupivacaine was administered along the RFA tract on removal of the probe.

The patient tolerated the procedure well and was discharged the next day. The histopathological examination showed an osteoid-forming lesion in loose fibrovascular stroma and prominent osteoblastic activity, confirming the diagnosis of OB. There was no evidence of bone permeation, severe atypia or atypical mitotic activity that would suggest malignancy. The patient's pain improved after the procedure and he was symptomfree during the six-month follow-up visit.

\section{DISCUSSION}

$\mathrm{OB}$ is a rare, osteoid-forming, benign primary neoplasm of the bone and is a close counterpart of osteoid osteoma. It is often called giant osteoid osteoma. OB accounts for approximately $1 \%$ of primary bone tumours and typically presents in young adults in the second-to-third decade of life, with a male-to-female ratio of 2:1. (1) OB can affect any bone, but it commonly arises from the vertebral column and long bones. When it arises from the vertebral column, $\mathrm{OB}$ has a predilection for the posterior 


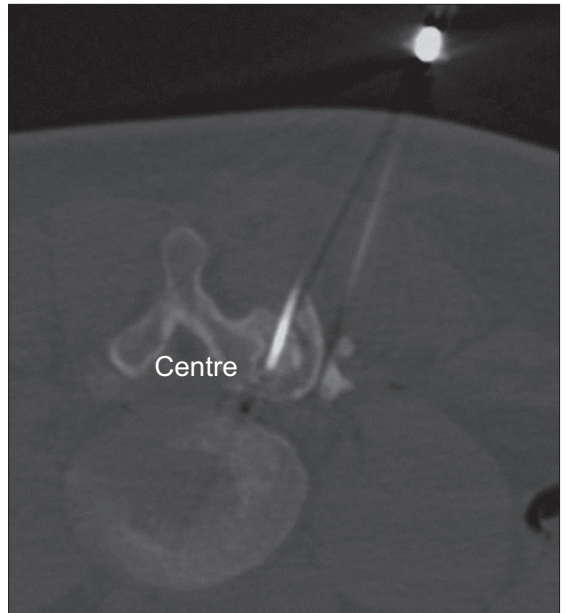

Fig. 4 Axial CT image shows the tip of the bone biopsy needle in the osteoblastoma.

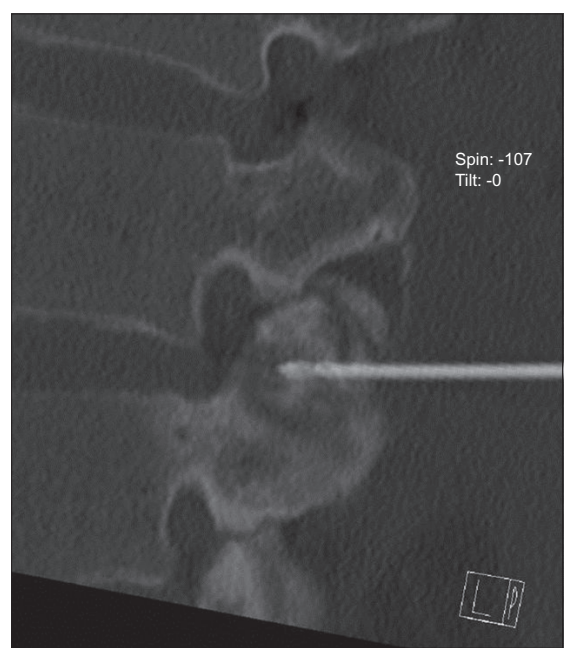

Fig. 5 Right parasagittal reformatted CT image shows the tip of the radiofrequency ablation probe in the osteoblastoma.

elements and can extend to involve the vertebral body. Within the long bones, OB commonly affects the diaphysis, followed by the metaphysis, and rarely the epiphysis. The most common location of osteoblastoma in the long bones is the proximal femur $(50 \%)$, followed by the tibia (19\%), humerus $(19 \%)$, radius $(7.7 \%)$ and fibula $(3.8 \%){ }^{(2)}$ The tumour is composed of fibroblasts and giant osteoclasts that produce osteoid and woven bone.

The most common presenting symptom of $\mathrm{OB}$ is pain, which is persistent and dull-aching in nature. Unlike in osteoid osteoma, the pain is neither worse at night nor relieved by salicylates. As the most common location of $\mathrm{OB}$ is the posterior elements of the vertebral column, patients can present with neurological symptoms due to compression or irritation of the adjacent nerve roots. ${ }^{(2)}$ Patients may also present with a deformity such as scoliosis or torticollis.

Imaging plays a vital role in the diagnosis of $\mathrm{OB}$. Radiographic features of $\mathrm{OB}$ can differ according to the type (i.e. simple or aggressive) and location of the lesion. It can present as a sclerotic or osteolytic expansile lesion with a central nidus and surrounding reactive bone changes. In young adults, an osteolytic lesion with central matrix mineralisation in the posterior elements of
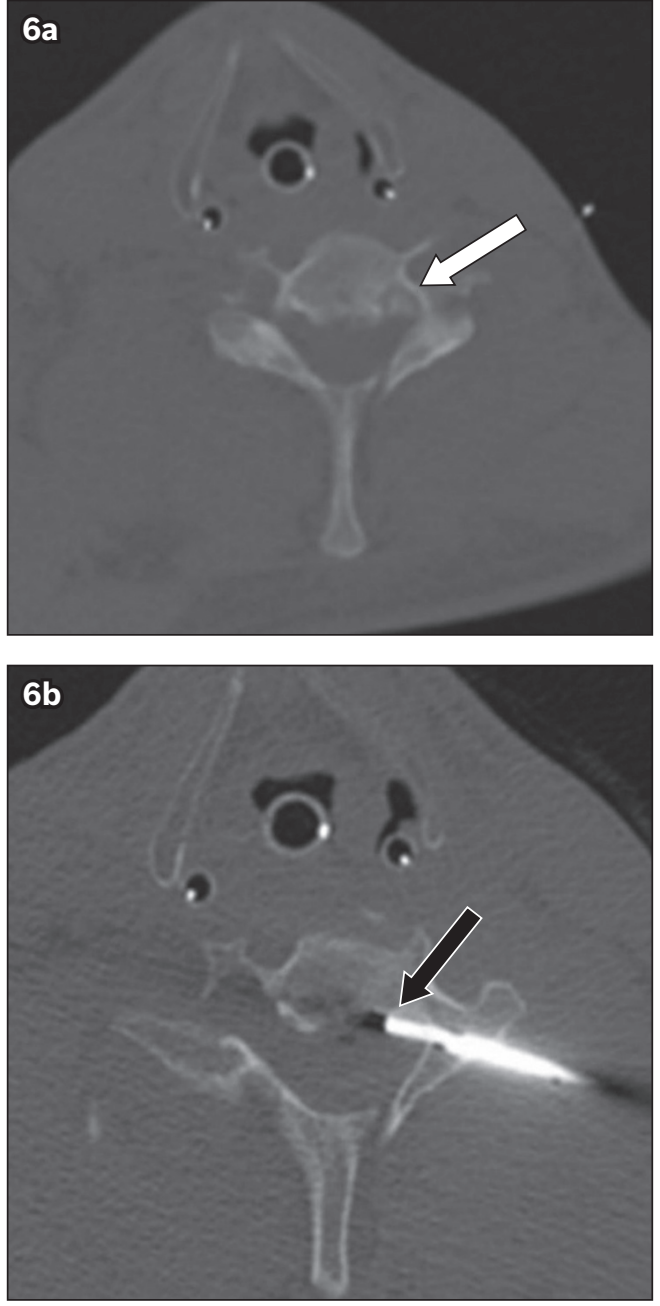

Fig. 6 Osteoid osteoma in a 40-year-old man who presented with neck pain at night. (a) Axial CT images of the C6 vertebra show (a) a small osteolytic lesion with central nidus (white arrow) in the left side of the vertebral body; and (b) the radiofrequency ablation probe in the nidus (black arrow).

the vertebral column is highly suggestive of $\mathrm{OB}$, particularly when the lesion is larger than $2 \mathrm{~cm}$. OB may sometimes have features of malignant lesions, such as thinning, erosion of cortex and associated soft-tissue mass. ${ }^{(3)} \mathrm{CT}$ is the imaging modality of choice, as it can accurately show the extent of the lesion, presence or absence of matrix mineralisation, location of the lesion (i.e. cortical or medullary) and surrounding bone changes. Furthermore, CT can also be used to guide biopsy and RFA of the lesion. ${ }^{(4)} \mathrm{MR}$ imaging is particularly useful for clear delineation of soft tissue and marrow oedema associated with the lesion, and to evaluate its mass effect on the spinal cord and the nerves. It is also useful for patient follow-up after RFA. The signal intensity of the lesion may vary with the degree of matrix mineralisation. MR imaging can, in rare instances, show fluid-fluid level within the lesion due to an aneurysmal bone cyst component. Bone scintigraphy often reveals a focus of increased radiotracer uptake, while SPECT/CT is helpful for accurate localisation of the lesion.

OB closely mimics osteoid osteoma. The typical features of osteoid osteoma are the relative stability and smaller size of the lesion $(<1 \mathrm{~cm})$, and pain that is worse at night and characteristically relieved by salicylates (Fig. 6a \& b). In contrast, OB may show non-characteristic pain, aggressive features, rapid 

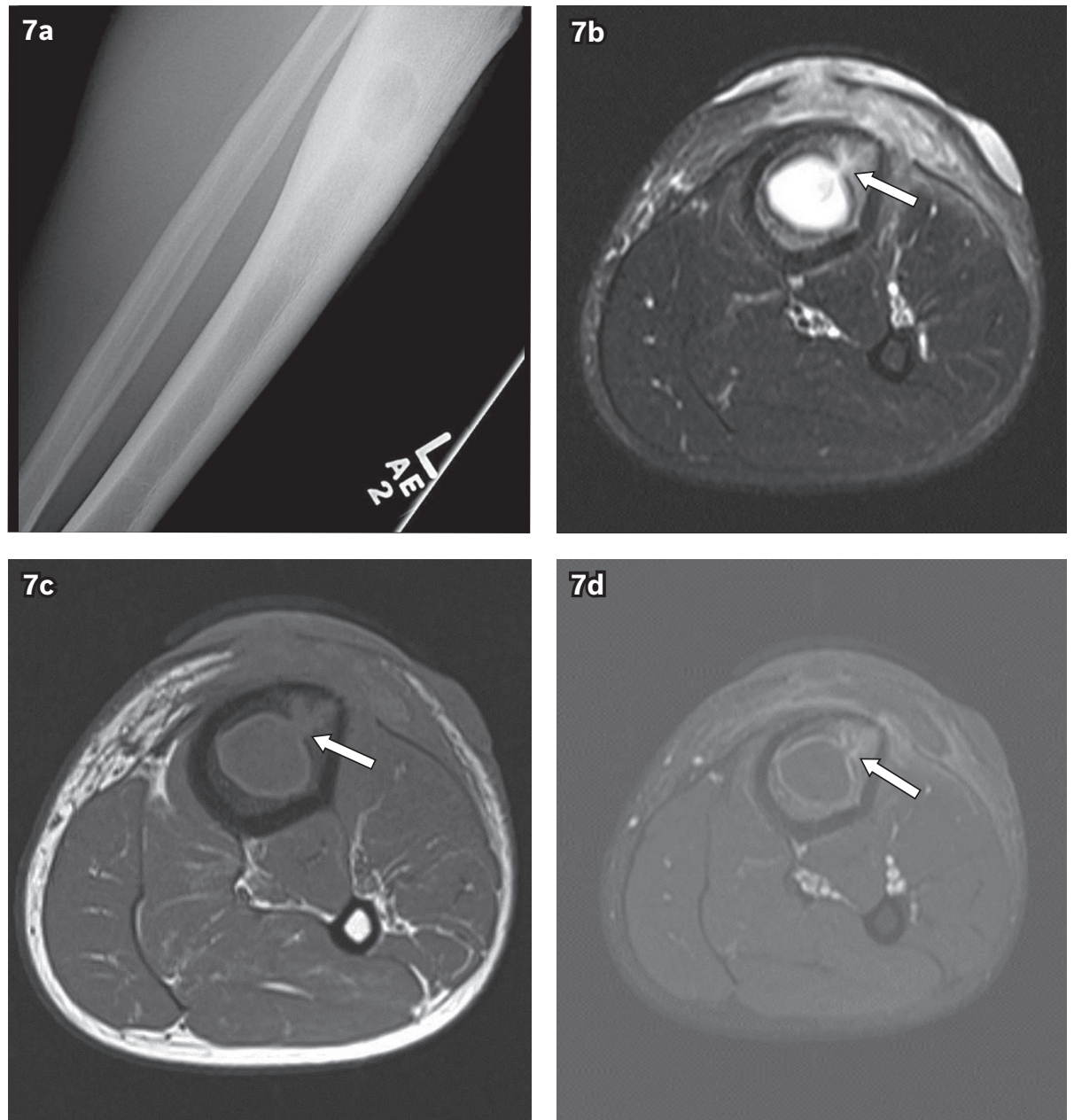

Fig. 7 Brodie's abscess in a 32-year-old man who presented with left leg pain. (a) Radiograph of the patient's left leg shows a well-defined osteolytic lesion with surrounding sclerosis in the proximal tibia. (b) T1-W turbo spin-echo (TSE), (c) T2-W TSE, and (d) contrast-enhanced T1-W TSE axial images of the upper left leg show a Brodie's abscess with overlying soft tissue changes; a tract (arrows) leads away from the abscess cavity.

growth and is usually larger in size $(>2 \mathrm{~cm})$. The sequestrum in a Brodie's abscess may resemble the nidus of OB; however, a tract leading away from the abscess cavity (Fig. 7a-d) is present in a Brodie's abscess and, hence, helps in differentiating between the two. ${ }^{(5)}$ Radiologically, it may be difficult to differentiate the aggressive form of $\mathrm{OB}$ from primary malignant bone tumours such as osteosarcoma. However, aggressive OB, which is very rare, tends to affect an older age group and have less periosteal reaction than in osteosarcoma. ${ }^{(6)}$ Histopathologically, large epithelioid osteoblasts and mild-to-moderate mitotic figures are characteristic features of aggressive OB. In contrast, an osteosarcoma has profuse mitotic figures and abundant osteoid. ${ }^{(6)}$ Secondary aneurysmal bone cyst (ABC) formation can sometimes occur in $\mathrm{OB}$; however, $\mathrm{OB}$ with secondary $\mathrm{ABC}$ will have some solid areas, unlike primary $\mathrm{ABC}$. $^{(6)}$

Treatment options for $\mathrm{OB}$ depend mainly on its size and location. RFA is preferred when the OB is small, lacks aggressive features and is located in long bones. The principle of RFA is the utilisation of thermal energy to destroy tumour cells and cause coagulation necrosis. The most important factors to consider before treating OB with RFA include dedicated CT-guided access planning, overlapping RFA needle positions within the $\mathrm{OB}$ nidus, multiple ablation and thermal protection for adjacent neural structures, if any. ${ }^{(7)} \mathrm{RFA}$ is performed at $90^{\circ} \mathrm{C}$ for a minimum of six minutes; for larger lesions, additional needle placement should be considered. In cases where a lack of intact cortex between the nidus and neural structures may allow propagation of radiofrequency energy and cause damage to adjacent nerves, neuroprotective measures such as insulation using an injection of air or carbon dioxide, dextrose infusion, or thermal monitoring with a suitable probe will enhance the safety of the procedure. ${ }^{(8)}$ Post-ablation syndrome, skin burn, damage to neural components, bleeding and infection are some known complications of RFA. Surgery is recommended when the $\mathrm{OB}$ is large and has aggressive features, as simple curettage alone has a high recurrence rate. Complete surgical resection of the $\mathrm{OB}$ is recommended. Bone grafting and internal fixation may be needed to repair the bone defect and prevent fracture. ${ }^{(9)}$ When neural compression is present, spinal decompression may be necessary.

In summary, $\mathrm{OB}$ is a rare, osteoid-forming, benign primary tumour of the bone, which commonly arises from the posterior elements of the vertebral column in young adults. The usual presenting symptom is a constant, dull-aching pain that is, typically, neither relieved by salicylates nor worse at night. CT is the imaging modality of choice. The imaging appearance of 
OB commonly mimics that of osteoid osteoma. However, it is usually larger in size ( $>2 \mathrm{~cm}$ ) and may have aggressive features. For the management of small, uncomplicated, benign-looking OBs, RFA is a simple and effective procedure.

ABSTRACT A 25-year-old man presented with chronic low back pain and occasional radiation to the right lower limb. Magnetic resonance imaging and computed tomography (CT) of the lumbar spine showed an osteolytic expansile lesion with a central sclerotic nidus in the right superior facet of the L5 vertebra and surrounding marrow oedema. The diagnosis of osteoblastoma was made based on imaging findings and confirmed after CT-guided biopsy. Radiofrequency ablation of the lesion was successfully performed. The patient tolerated the procedure well and showed symptomatic relief. The imaging features and management of osteoblastoma are discussed.

Keywords: computed tomography, magnetic resonance imaging, osteoblastoma, radiofrequency ablation

\section{REFERENCES}

1. Lucas DR, Unni KK, McLeod RA, O'Connor MI, Sim FH. Osteoblastoma: clinicopathologic study of 306 cases. Hum Pathol 1994; 25:117-34.

2. Kroon HM, Schurmans J. Osteoblastoma: clinical and radiologic findings in 98 new cases. Radiology 1990; 175:783-90.

3. González-Sistal A, Baltasar Sánchez A. A complementary method for the detection of osteoblastic metastases on digitized radiographs. J Digit Imaging 2006; 19:270-5.

4. Rehnitz C, Sprengel SD, Lehner B, et al. CT-guided radiofrequency ablation of osteoid osteoma and osteoblastoma: clinical success and long-term follow up in 77 patients. Eur J Radiol 2012; 81:3426-34.

5. Greenspan A, Jundt G, Remagen W. Bone forming (osteogenic) lesions. In: Differential diagnosis in orthopaedic oncology. 2nd ed. Philadelphia: Lippincott Williams \& Wilkins 2006: 40-157.

6. Singh DK, Das KK, Mehrotra A, et al. Aggressive osteoblastoma involving the craniovertebral junction: A case report and review of literature. J Craniovertebr Junction Spine 2013; 4:69-72.

7. Weber MA, Sprengel SD, Omlor GW, et al. Clinical long-term outcome, technical success, and cost analysis of radiofrequency ablation for the treatment of osteoblastomas and spinal osteoid osteomas in comparison to open surgical resection. Skeletal Radiol 2015; 44:981-93.

8. Rybak LD, Gangi A, Buy X, La Rocca Vieira R, Wittig J. Thermal ablation of spinal osteoid osteomas close to neural elements: technical considerations. AJR Am J Roentgenol 2010; 195:W293-8.

9. Li Z, Zhao Y, Hou S, et al. Clinical features and surgical management of spinal osteoblastoma: a retrospective study in 18 cases. PLoS One 2013; 8:e74635. 


\section{SINGAPORE MEDICAL COUNCIL CATEGORY 3B CME PROGRAMME} (Code SMJ 201702B)

Question 1. Regarding osteoblastoma:

(a) It is an osteoid-forming, benign primary neoplasm.

(b) It accounts for approximately $5 \%$ of primary bone tumours.

(c) Within the long bones, it commonly affects the metaphysis.

(d) Posterior elements of the vertebral column are one of the common sites.

Question 2. Regarding clinical presentations of osteoblastoma:

(a) It causes persistent and dull-aching pain.

(b) The pain is characteristically worse at night and relieved by salicylates.

(c) Patients can present with neurological symptoms.

(d) Patients can present with painful scoliosis.

Question 3. Regarding imaging of osteoblastoma:

(a) An osteolytic lesion with matrix mineralisation that is less than $1 \mathrm{~cm}$ is a characteristic feature.

(b) Computed tomography is the imaging modality of choice.

(c) Magnetic resonance imaging is particularly useful to define the involvement of soft tissue and neural elements.

(d) Bone scintigraphy can help to detect osteoblastic activity.

Question 4. Regarding diagnosis and differential diagnosis of osteoblastoma:

(a) Osteoblastoma is relatively stable when compared to osteoid osteoma.

(b) A tract leading away from the abscess cavity is helpful in differentiating a Brodie's abscess from an osteoblastoma.

(c) There is more periosteal reaction in osteosarcomas as compared to aggressive osteoblastomas.

(d) Epithelioid osteoblast is a characteristic histologic feature of aggressive osteoblastomas.

Question 5. Regarding management of osteoblastoma:

(a) Radiofrequency ablation (RFA) is preferred for small, uncomplicated osteoblastoma in long bones.

(b) RFA is performed at $90^{\circ} \mathrm{C}$ for a maximum of two minutes.

(c) Additional RFA needle placements may be needed in larger lesions.

(d) Simple curettage alone has a high recurrence rate.

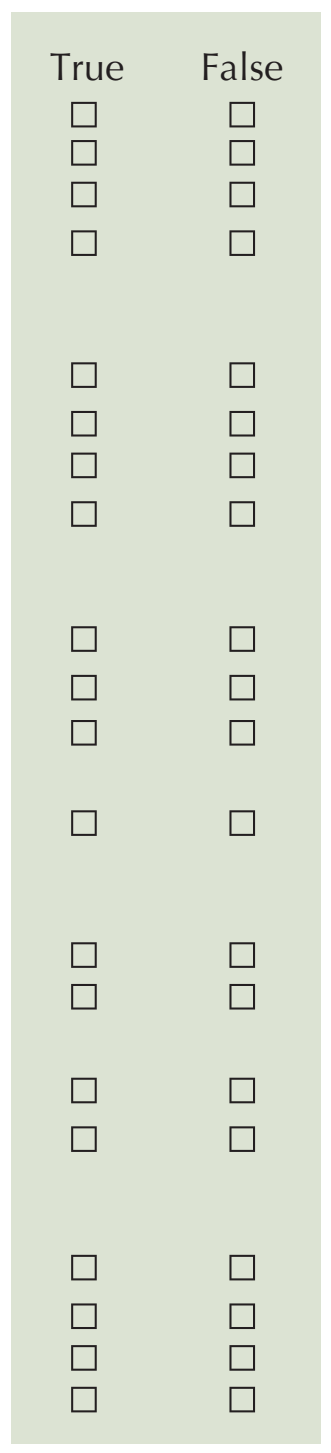

\section{Doctor's particulars:}

Name in full

MCR number

Email address

\section{SUBMISSION INSTRUCTIONS:}

(1) Visit the SMJ website: http://www.smj.org.sg/current-issue and select the appropriate set of questions. (2) Provide your name, email address and MCR number. (3) Select your answers and click "Submit".

\section{RESULTS:}

(1) Answers will be published online in the SMJ April 2017 issue. (2) The MCR numbers of successful candidates will be posted online at the SMJ website by 30 March 2017. (3) Passing mark is $60 \%$. No mark will be deducted for incorrect answers. (4) The SMJ editorial office will submit the list of successful candidates to the Singapore Medical Council. (5) One CME point is awarded for successful candidates.

Deadline for submission: (February 2017 SMJ 3B CME programme): 12 noon, 23 March 2017. 